\title{
Involvement of miR-9/MCPIP1 axis in PDGF-BB-mediated neurogenesis in neuronal progenitor cells
}

\author{
L Yang ${ }^{1,2}, \mathrm{~J} \mathrm{Chao}^{1,2}, \mathrm{YH} \mathrm{Kook}^{1}, \mathrm{Y} \mathrm{Gao}^{1}, \mathrm{H}$ Yao ${ }^{1}$ and SJ Buch ${ }^{*, 1}$
}

Highly conserved microRNA-9 (miR-9) has a critical role in various cellular processes including neurogenesis. However, its regulation by neurotropins that are known to mediate neurogenesis remains poorly defined. In this study, we identify plateletderived growth factor-BB (PDGF-BB)-mediated upregulation of miR-9, which in turn downregulates its target gene monocyte chemotactic protein-induced protein 1 (MCPIP1), as a key player in modulating proliferation, neuronal differentiation as well as migration of neuronal progenitor cells (NPCs). Results indicate that miR-9-mediated NPC proliferation and neuronal differentiation involves signaling via the nuclear factor-kappa B (NF- $\kappa \mathrm{B}$ ) and CAMP response element-binding protein (CREB) pathways, and that NPC migration involves CREB but not the NF- $\kappa$ B signaling. These findings thus suggest that miR-9-mediated downregulation of MCPIP1 acts as a molecular switch regulation of neurogenesis.

Cell Death and Disease (2013) 4, e960; doi:10.1038/cddis.2013.486; published online 12 December 2013

Subject Category: Neuroscience

New dentate granule cells are continuously generated from neural progenitor cells and are integrated into the existing hippocampal circuitry in the adult mammalian brain by a process termed as neurogenesis. ${ }^{1}$ Neurotropic family of growth factors have key roles in maintaining neuronal homeostasis via regulation of neurogenesis. ${ }^{2,3}$ Growth factors belonging to the platelet-derived growth factor (PDGF) family are composed of products of four gene products $(A-D)$ that can dimerize and bind to two receptor tyrosine kinases, PDGF- $\alpha \mathrm{R}$ and $-\beta \mathrm{R}^{4-6}$ Our previous study has implicated PDGF-BB as a crucial factor in the regulation of neuronal progenitor cell (NPC) proliferation. ${ }^{7}$

In keeping with the emerging interest in the molecular mechanisms controlling neurogenesis in the central nervous system (CNS), a new and exciting aspect of gene regulation has gained attention in recent years with the discovery of mammalian microRNAs (miRNAs). ${ }^{8-10}$ miRNAs are small, evolutionarily conserved noncoding RNAs that are derived from much larger primary transcripts. MicroRNA-9 (miR-9), a highly conserved microRNA, is expressed predominantly in the CNS of the developing embryo exhibiting a prodifferentiation function embryo. ${ }^{11-15}$ MiR-9 has been shown to regulate axonal extension and branching via targeting Map1b in mouse cortical neurons. ${ }^{16}$ Moreover, recent studies have also implicated the role of miR-9 in controlling stability of the Notch target mRNA-Hes1 that is critical for Hes1 ultradian oscillations. ${ }^{17}$ Although miR-9 has been documented to promote proliferation of neuronal progenitors by targeting stathimin, ${ }^{12}$ whether miR-9 has a role in growth factor (PDGF-BB)mediated regulation of neurogenesis is not well understood.

Using computational algorithms such as the TargetScan, monocyte chemotactic protein-induced protein 1 (MCPIP1) gene $(Z C 3 H 12 A)$, known to control inflammation ${ }^{18,19}$ was identified as the predicted target of miR-9. MCPIP1 has been identified to promote glial differentiation in NT2 neuroprogenitor cells treated with monocyte chemotactic protein-1 (MCP-1). ${ }^{20}$ Furthermore, it has also been implicated as a negative regulator of macrophage activation. ${ }^{19}$ Whether MCPIP1 has a role in PDGF-BB-mediated regulation of neurogenesis however remains an enigma.

In the present study, we sought to understand the signaling pathways involved in PDGF-BB-mediated regulation of NPC proliferation, neuronal differentiation and migration with the involvement of miR-9 and its targeted suppression of MCPIP1. We further demonstrated distinct downstream activation of the nuclear factor-kappa B (NF- $k$ B) in NPC proliferation and differentiation while activating the CAMP response element-binding protein (CREB) pathway in all of the neurogenesis processes.

\section{Results}

PDGF-BB-mediated upregulation of miR-9 in NPCs. Our previous study demonstrated that PDGF-BB has a critical role in the regulation of NPC proliferation. ${ }^{7}$ In addition, miR-9 has also been implicated in NPC proliferation and migration. ${ }^{12}$

\footnotetext{
1Department of Pharmacology and Experimental Neuroscience, University of Nebraska Medical Center, Omaha, NE 68198-5880, USA

${ }^{*}$ Corresponding author: SJ Buch, Department of Pharmacology and Experimental Neuroscience, 985880 Nebraska Medical Center (DRC 8011$)$, University of Nebraska Medical Center, Omaha, NE 68198-5880, USA. Tel: +1 402559 3165; Fax: +1 402559 3744; E-mail: sbuch@unmc.edu

${ }^{2}$ These authors contributed equally to this work.

Keywords: neuronal progenitor cell; PDGF-BB; miR-9; MCPIP1; neurogenesis

Abbreviations: PDGF, platelet-derived growth factor; CNS, central nervous system; NPC, neuronal progenitor cell; miRNAs, microRNAs; miR-9, microRNA-9; MCPIP1, monocyte chemotactic protein-induced protein 1; MCP-1, monocyte chemotactic protein-1; NF- $\kappa$ B, nuclear factor-kappa B; CREB, cAMP response elementbinding protein; FISH, fluorescence in situ hybridization; GFAP, glial fibrillary acidic protein; 3-D, 3-dimensional; UTR, untranslated region; OE, overexpressing; ERK, extracellular signal-regulated protein kinase; PI3K, phosphatidylinositol 3-kinase; MEK, mitogen-activated protein kinase; PKA, protein kinase A; PBS, phosphatebuffered saline; RT, room temperature; WT, wild type; SSC, saline-sodium citrate; S.D., standard deviation

Received 02.10.13; revised 04.11.13; accepted 05.11.13; Edited by A Verkhratsky
} 
However, very little is known about the mechanism by which PDGF-BB exerts its action through miR-9. In the present study, NPCs were exposed to PDGF-BB $(100 \mathrm{ng} / \mathrm{ml})$ for varying time points. As an initial screen to identify which of the miR-9 precursors were regulated in response to PDGF-BB, mRNA levels of miR-9-1, miR-9-2 and miR-9-3 were determined by real time-PCR. Compared with the expression of pre-miR-9-1 (Figure 1a), miR-9-2 mRNA was upregulated (2.36- and 4.08-fold) at 6 and $12 \mathrm{~h}$, respectively (Figure $1 \mathrm{~b}$ ). The level of miR-9-3 was not detectable (data not shown). Consistent with this finding and as shown in Figure 1c, NPCs cultured in the presence of PDGF-BB demonstrated upregulation of mature miR-9 in a time-dependent manner. Having determined that PDGF-BB mediated increased expression of miR-9 by real-time PCR, the next step was to confirm the increased miR-9 expression by using fluorescence in situ hybridization (FISH). Compared with control, PDGF-BB treatment resulted in increased miR-9 expression in NPCs (Figure 1d). Cumulatively, these data clearly demonstrate PDGF-BB-mediated induction of miR-9 in NPCs.
Involvement of miR-9 in PDGF-BB-mediated NPC proliferation, differentiation and migration. As miR-9 expression was increased in the NPCs in the presence of PDGF-BB, we next sought to explore the role of miR-9 in PDGF-BB-mediated NPC proliferation, differentiation and migration. NPCs were transduced with the lentivirus-expressing anti-miR-9 and assessed for cell proliferation. As shown in Figure 2a, PDGF-BB increased NPC proliferation, which was significantly inhibited by anti-miR-9.

Newly generated neural cells undergo differentiation before they mature and become functional. Having determined the role of PDGF-BB in proliferation, it was of interest to investigate the role of miR-9 in the differentiation of NPCs. As shown in Figure $2 b$, exposure of NPCs to PDGF-BB resulted in a time-dependent increase in the expression of the neuronal marker ( $\beta$-III-tubulin), with a concomitant decrease in the expression of astrocyte marker (glial fibrillary acidic protein, GFAP), indicating thereby that PDGF-BB stimulated neuronal differentiation while inhibiting astrocyte differentiation. This was further confirmed by immunostaining the cells with
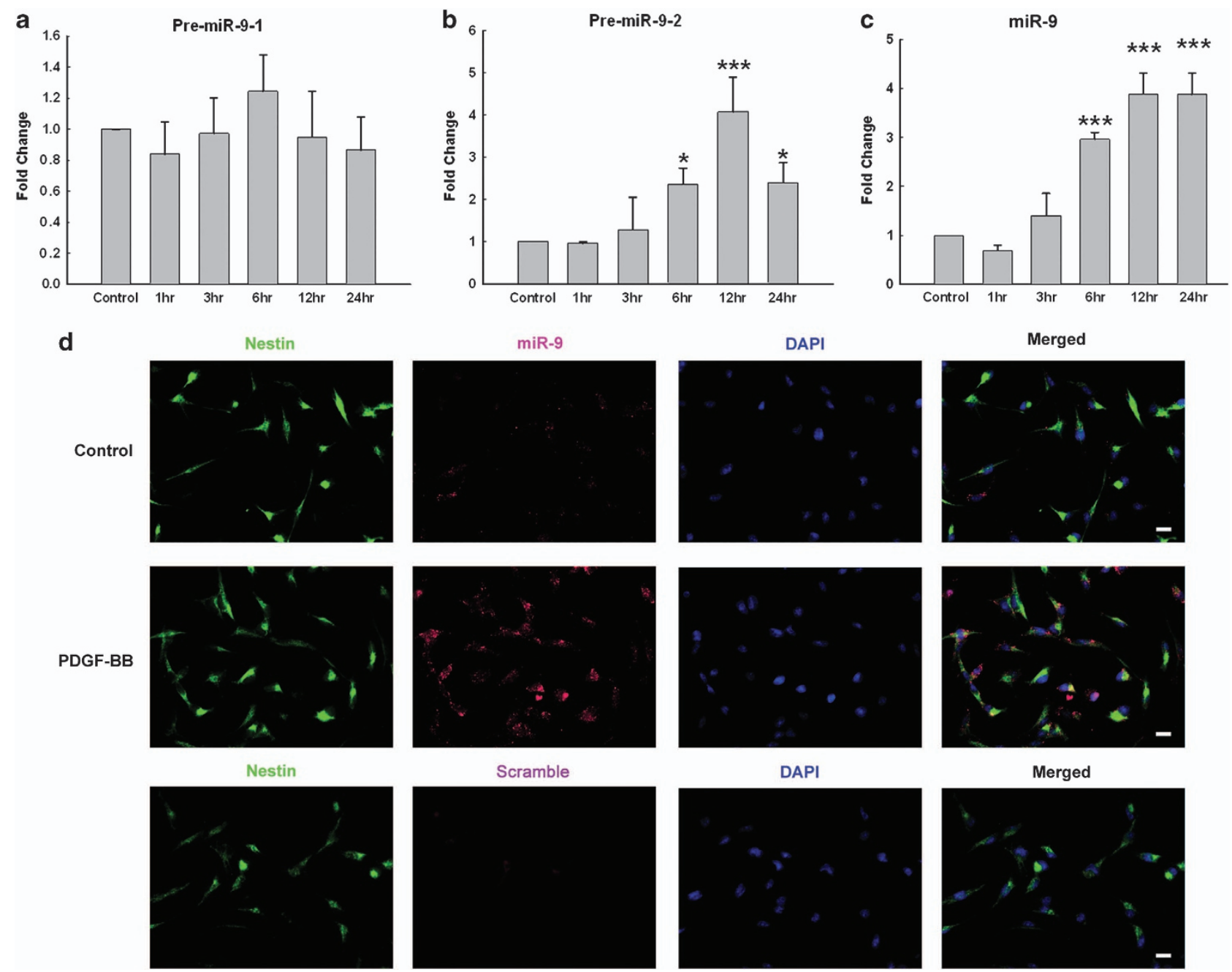

Figure 1 PDGF-BB-mediated upregulation of miR-9 in NPCs. Total RNA isolated from NPCs was subjected to real-time PCR analysis using primer sets specific for pre-miR-9-1 (a), pre-miR-9-2 (b) and mature miR-9 (c). PDGF-BB markedly upregulated pre-miR-9-2 mRNA expression compared with the expression of pre-miR-9-1. (d) FISH analysis of mature miR-9 in primary NPCs. Nestin: green; miR-9: red; 4',6-diamidino-2-phenylindole (DAPI): blue. Scale bar $=5 \mu \mathrm{m}$. All the data are indicated as mean \pm S.D. of four individual experiments. ${ }^{\star} P<0.05,{ }^{\star \star \star} P<0.001$ versus control group 
a

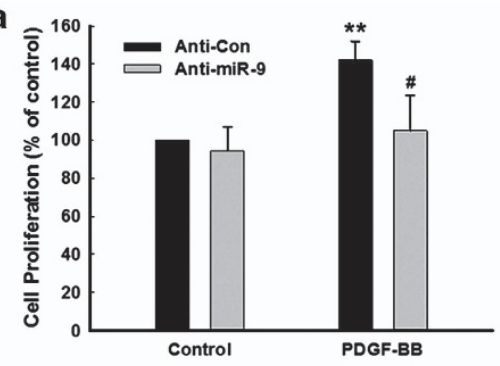

b

PDGF-BB (day) $\quad \begin{array}{lllll}0 & 1 & 3 & 5 & 7\end{array}$
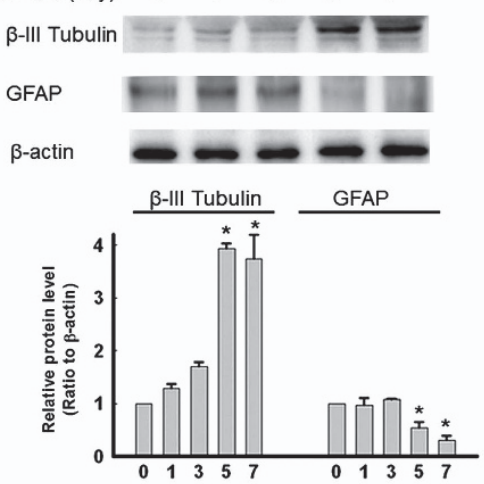

c
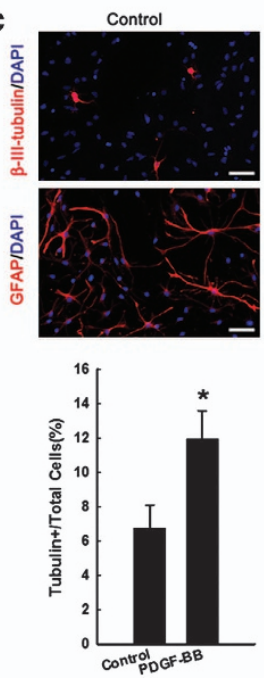

PDGF-BB
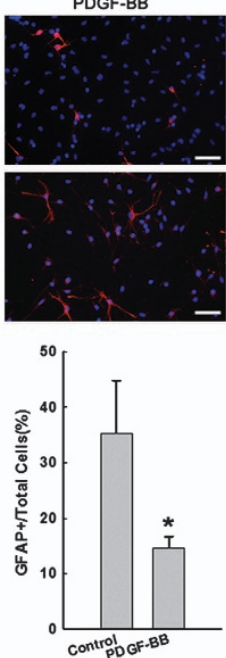

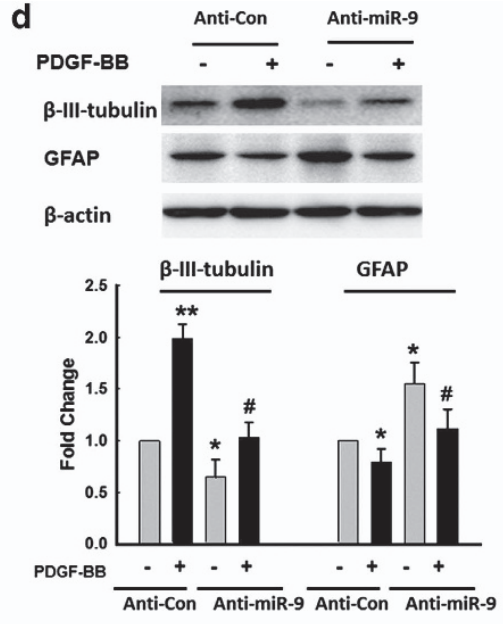

e

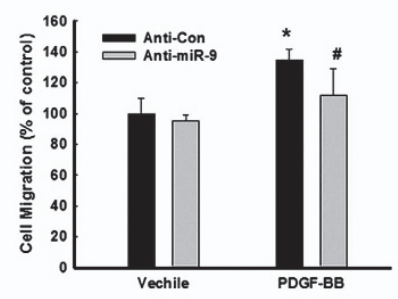

f
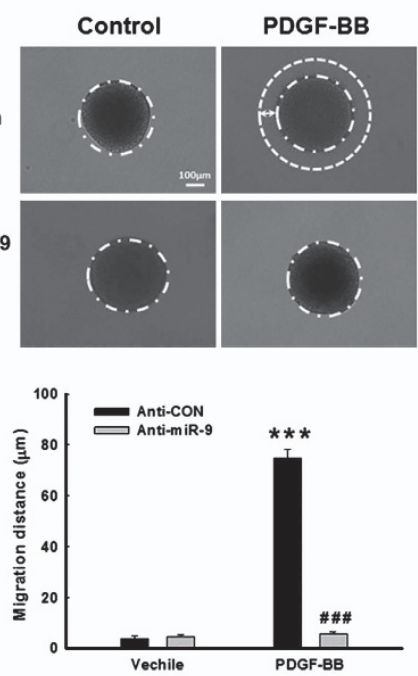

Figure 2 Involvement of miR-9 in PDGF-BB-mediated NPC proliferation, differentiation and migration. (a) Transduction of primary NPCs with anti-miR-9 precursor resulted in amelioration of PDGF-BB-mediated increase in NPC proliferation. (b) PDGF-BB increased neuronal while decreasing astrocyte differentiation as demonstrated by western blotting (WB) analysis. Lysates of NPCs exposed to PDGF-BB for 0-7days were assessed for neuronal and astrocyte-specific markers such as $\beta$-III-tubulin and GFAP, respectively, by WB analysis. Representative figures are shown from four individual experiments. (c) Immunostaining of $\beta$-III-tubulin and GFAP in NPCs. Representative images of $\beta$-III-tubulin- (upper panel) and GFAP- (lower panel) positive cells in NPCs. Scale bar $=20 \mu \mathrm{m}$. (d) Transduction of primary NPCs with anti-miR-9 resulted in abrogation of PDGF-BB-mediated increase in neuronal differentiation and a concomitantly opposing effect on GFAP expression. (e) Transduction of primary NPCs with anti-miR-9 resulted in decreased NPC migration induced by PDGF-BB using Boyden chamber. (f) Transduction of primary NPCs with anti-miR-9 resulted in decreased NPC migration induced by PDGF-BB using the $3-D$ cell culture system. All the data are indicated as mean \pm S.D. of four individual experiments. ${ }^{*} P<0.05$, ${ }^{* *} P<0.01$, ${ }^{* * *} P<0.001$ versus control group or NPCs transduced with anti-control lentivirus in control group; ${ }^{\#} P<0.05,{ }^{\# \# \#} P<0.001$ versus NPCs transduced with anti-control lentivirus in PDGF-BB-treated group

the respective cell-specific markers (Figure 2c). Next, we sought to examine the role of miR-9 in PDGF-BB-mediated differentiation of NPCs. As shown in Figure 2d, transduction of NPCs with anti-miR-9 resulted in decreased expression of $\beta$-III-tubulin with a concomitant increase in the expression of GFAP compared with cells that were transduced with the
anti-miR-control in the presence of PDGF-BB. These findings thus suggest the involvement of miR-9 in PDGF-BB-mediated neuronal differentiation.

In addition to NPC proliferation and differentiation, NPC migration is also a critical aspect of neurogenesis. We next sought to explore the role of miR-9 in PDGF-BB-mediated migration. 
Using Boyden chambers, it was demonstrated that there was increased migration of NPCs in the presence of PDGF-BB (Figure 2e). Intriguingly, transduction of cells with anti-miR-9 lentivirus resulted in inhibition of PDGF-BBmediated induction of NPC migration, thus underpinning the role of miR-9 in the process. Further validation of these findings was carried out by monitoring NPC migration in a 3-dimensional (3-D) cell culture system. As shown in Figure $2 f$, there was increased migration of NPCs in response to PDGF-BB and this effect was inhibited in cells transduced with anti-miR-9 compared with anti-miR control vector.

PDGF-BB-mediated downregulation of MCPIP1 in NPCs. Having determined the role of miR-9 in PDGF-BB-mediated NPC proliferation, differentiation and migration, we next sought to investigate the mechanism(s) underlying these effects. MCPIP1, a $\mathrm{CCCH}$ zinc-finger ribonuclease (ZC3H12A) with conserved miR-9 binding site within its $3^{\prime}$-untranslated region (UTR) has been predicted as the putative target of miR-9 using the TargetScan algorithm. However, whether MCPIP1 was regulated by PDGF-BB remains poorly understood. We thus initially examined the effect of PDGF-BB on the transcription of MCPIP1. As shown in Figure 3a, following PDGF-BB exposure, MCPIP1 mRNA was time-dependently downregulated from 3 to $24 \mathrm{~h}$. To further examine whether the mRNA downregulation translated into decreased protein expression, the MCPIP1 expression at the protein level was examined. As shown in Figure 3b, PDGF-BB downregulated MCPIP1 protein expression in a time-dependent manner. Interestingly, PDGF-BBinduced regulation of miR-9 correlated inversely with the expression of MCPIP1. These data thus suggested that PDGF-BB via upregulation of miR-9 mediated downregulation of MCPIP1 mRNA and protein in NPCs.

Involvement of MCPIP1 in PDGF-BB-mediated NPC proliferation, differentiation and migration. Having determined the MCPIP1 expression was downregulated in the NPCs in the presence of PDGF-BB, we next wanted to assess the role of MCPIP1 in PDGF-BB-mediated NPC proliferation, differentiation and migration. To determine whether miR-9-mediated functional effects depend specifically on MCPIP1 suppression, an expression construct encoding the entire MCPIP1 coding sequence but lacking the $3^{\prime}$-UTR, yielding an mRNA resistant to miRNA-mediated suppression was generated. NPCs were transduced with the MCPIP1 construct lacking the UTRs (MCPIP1- $\Delta 3^{\prime}-U T R$ ) that was not targeted by miR-9. To examine the involvement of the MCPIP1 in PDGF-BB-mediated NPC proliferation, cells were transfected with either vector control or MCPIP1 overexpressing (OE)- $\Delta 3^{\prime}-$ UTR constructs followed by exposure of cells to PDGF-BB. Intriguingly, PDGF-BB-mediated increase of NPC proliferation was significantly inhibited in cells transduced with the MCPIP1 OE - $\Delta 3^{\prime}-$ UTR construct, but not in cells transduced with the control vector (Figure $4 \mathrm{a}$ ). Collectively, these findings underscore the role of MCPIP1 in PDGF-BB-mediated increase of NPC proliferation.

Next, we examined the role of MCPIP1 in PDGF-BBmediated NPC differentiation. As shown in Figure 4b, transfection of NPCs with MCPIP1 OE- $\Delta 3^{\prime}-$ UTR construct

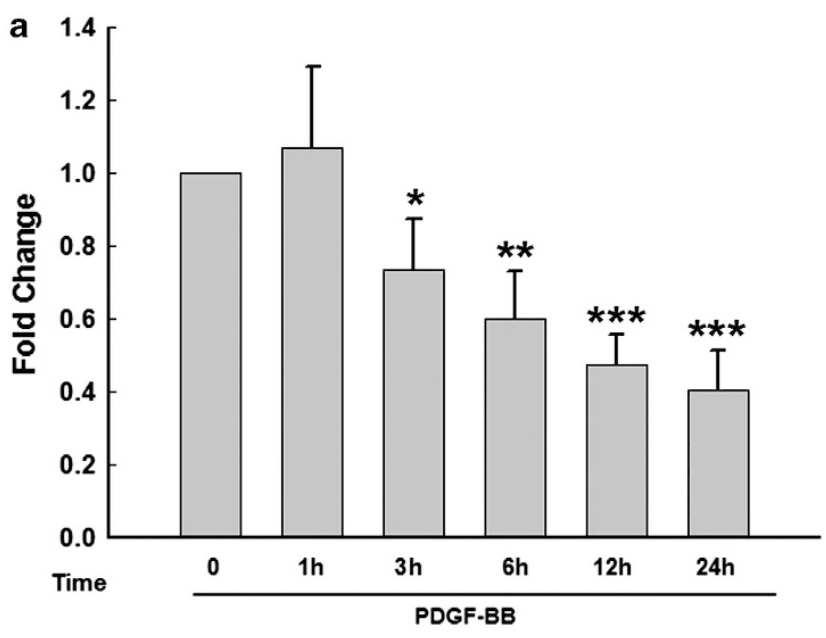

b
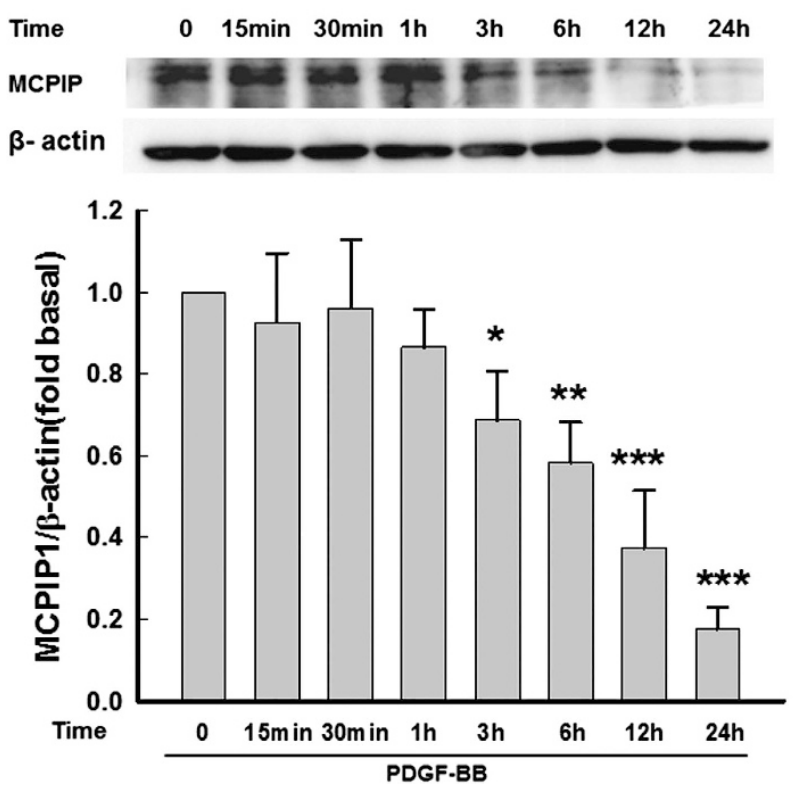

Figure 3 PDGF-BB-mediated downregulation of MCPIP1 in NPCs. (a) Total RNA isolated from NPCs was subjected to real-time PCR analysis using primer sets specific for MCPIP1. PDGF-BB markedly downregulated MCPIP1 mRNA expression compared with control NPCs. (b) Whole-cell lysates from PDGF-BBexposed cells were subjected to western blotting (WB) analysis using antibodies specific for MCPIP1. PDGF-BB exposure resulted in a time-dependent decrease in MCPIP1 protein in NPCs. All the data are presented from three individual experiments. All the data are indicated as mean \pm S.D. of four individual experiments. ${ }^{*} P<0.05,{ }^{* *} P<0.01$ and ${ }^{* * *} P<0.001$ versus control group

resulted in decreased expression of $\beta$-III-tubulin with a concomitant increase in the expression of GFAP compared with cells transduced with vector control, following treatment with PDGF-BB. The next step was to dissect the role of MCPIP1 in NPC migration in response to PDGF-BB. As shown in Figures $4 \mathrm{c}$ and $\mathrm{d}$, transfection of NPCs with MCPIP1 OE- $\Delta 3^{\prime}-U T R$ construct resulted in significant abrogation of PDGF-BB-mediated NPC migration in both Boyden chamber and 3-D matrix migration assays.

MCPIP1 overexpression antagonizes the effects of miR-9. To examine whether the miR-9/MCPIP1 interaction 

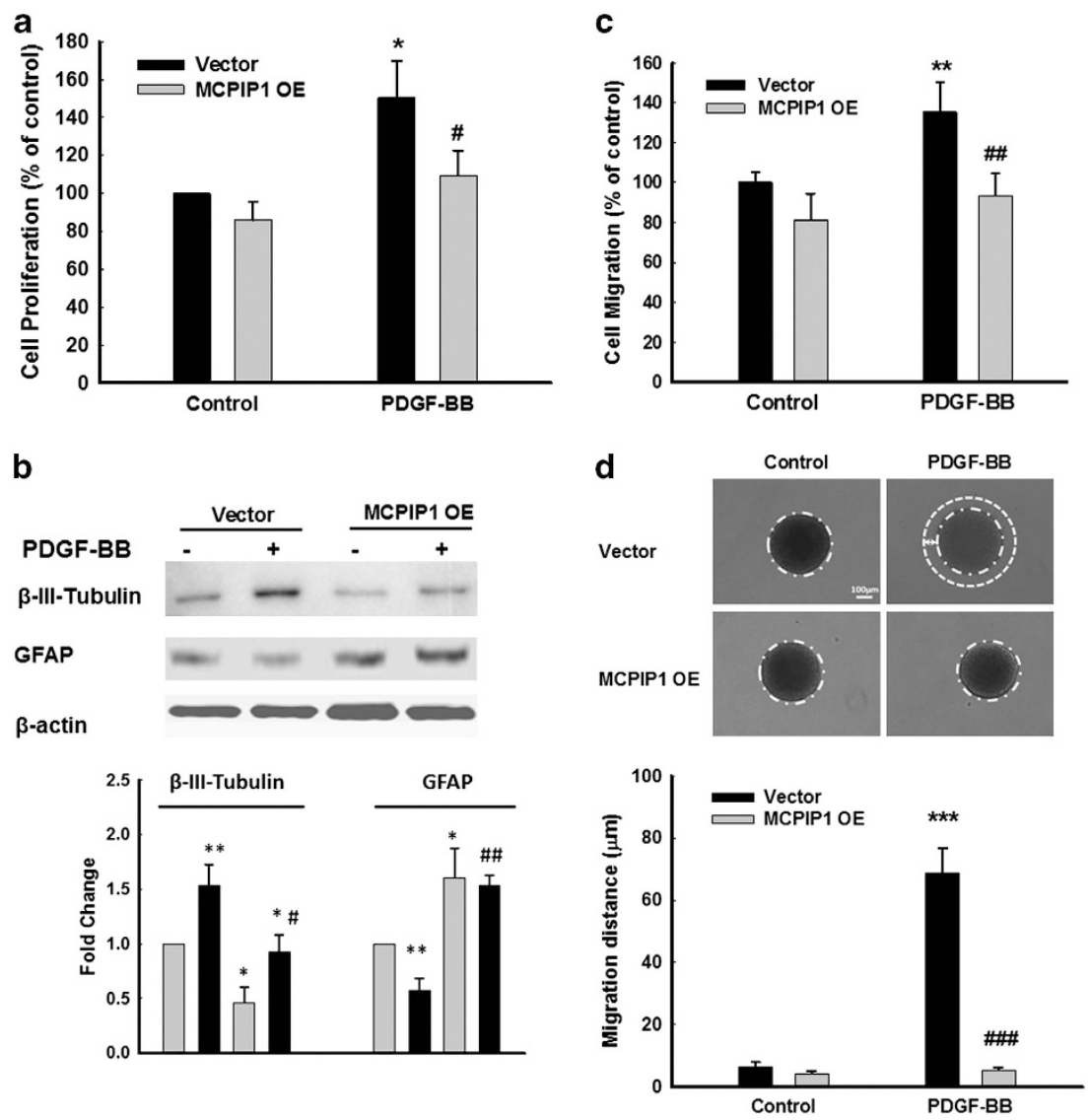

Figure 4 Involvement of MCPIP1 in PDGF-BB-mediated NPC proliferation, differentiation and migration. (a) Transfection of primary NPCs with MCPIP1 OE-3'-UTR construct resulted in amelioration of PDGF-BB-mediated increase in NPC proliferation. (b) Transfection of primary NPCs with MCPIP1 OE-3'-UTR construct resulted in abrogation of PDGF-BB-mediated increase of neuronal differentiation and a concomitantly opposing effect on GFAP expression. (c) Transfection of primary NPCs with MCPIP1 OE-3'-UTR construct resulted in abrogation of PDGF-BB-mediated upregulation of migration using the Boyden chamber. (d) Transfection of primary NPCs with MCPIP1 OE-3'-UTR construct resulted in abrogation of PDGF-BB-mediated upregulation of migration using the 3-D cell culture system. All the data are indicated as mean \pm S.D. of four individual experiments. ${ }^{\star} P<0.05,{ }^{\star \star} P<0.01,{ }^{* \star \star} P<0.001$ versus NPCs transfected with vector in control group; ${ }^{\#} P<0.05$, ${ }^{\# \#} P<0.01$, ${ }^{\# \# \#} P<0.001$ versus NPCs transfected with vector in PDGF-BB-treated group

modulates NPC proliferation, cells transfected with the MCPIP1 OE- $\Delta 3^{\prime}$-UTR construct were assessed for proliferation. Interestingly, there was a failure of miR-9 to induce NPC proliferation in cells transfected with the MCPIP1 OE- $\Delta 3^{\prime}$ UTR construct (Figure 5a). Similarly, while miR-9 enhanced neuronal differentiation, this effect was significantly suppressed in NPCs transfected with MCPIP1 OE- $\Delta 3^{\prime}$-UTR construct (Figure $5 \mathrm{~b}$ ). Furthermore, while transduction of miR-9 promoted migration of NPCs in Boyden chamber (Figure $5 c$ ) as well as in 3-D migration assay (Figure $5 d$ ), transfection of cells with MCPIP1 OE- $\Delta 3^{\prime}$-UTR construct abrogated this effect. These findings thus indicate that MCPIP1 is a key downstream target of miR-9.

MCPIP1 overexpression attenuates miR-9-mediated signaling. Having determined that miR-9 and its target MCPIP1 regulated NPC proliferation, differentiation and migration, we next sought to determine the intracellular signaling pathways involved in these processes mediated by miR-9/MCPIP1. It has been previously demonstrated that both the extracellular signal-regulated protein kinase (ERK) and Akt and the downstream transcription factors NF- $\kappa \mathrm{B}$ and
CREB have crucial roles in PDGF-BB-mediated proliferation of NPCs. Extension of these studies to decipher the role of MCPIP1 in miR-9-mediated activation of ERK and Akt in NPCs. In the presence of miR-9, there was increased activation of both the signaling mediators (ERK and Akt), and this effect was abrogated in cells transfected with MCPIP1 OE- $\Delta 3^{\prime}-$ UTR construct (Figure 6a). The next step was to investigate the role of MCPIP1 in miR-9-mediated activation of downstream transcription factors (NF- $\kappa \mathrm{B}$ \& CREB) in NPCs. Cells transduced with miR-9 exhibited increased activation and nuclear translocation of NF- $\kappa \mathrm{B}$ and CREB, and reciprocally, overexpression of MCPIP1 attenuated this effect (Figure 6b). Next logical step then was to examine whether there existed a link that could tie together the activation of ERK and phosphatidylinositol 3-kinase $(\mathrm{PI} 3 \mathrm{~K}) / \mathrm{Akt}$ with $\mathrm{NF}-\kappa \mathrm{B}$ and CREB. NPCs were pretreated with mitogen-activated protein kinase (MEK), or PI3K inhibitors followed by treatment with PDGF-BB and assessed for expression of NF- $\kappa$ B or CREB. As shown in Figure $6 \mathrm{c}$, both MEK and PI3K inhibitors significantly inhibited PDGFBB-mediated NF- $\kappa$ B activation. Similar to NF- $\kappa$ B, CREB activation was also dependent on ERK activation. 


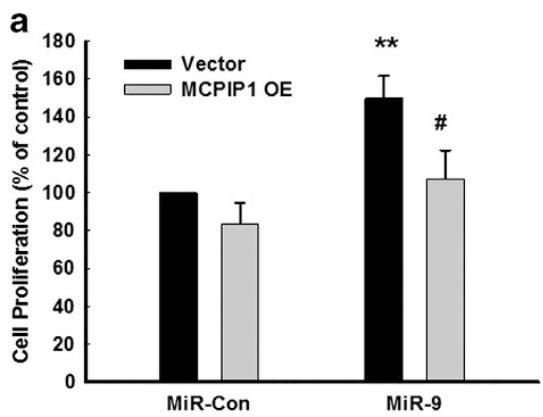

C

b
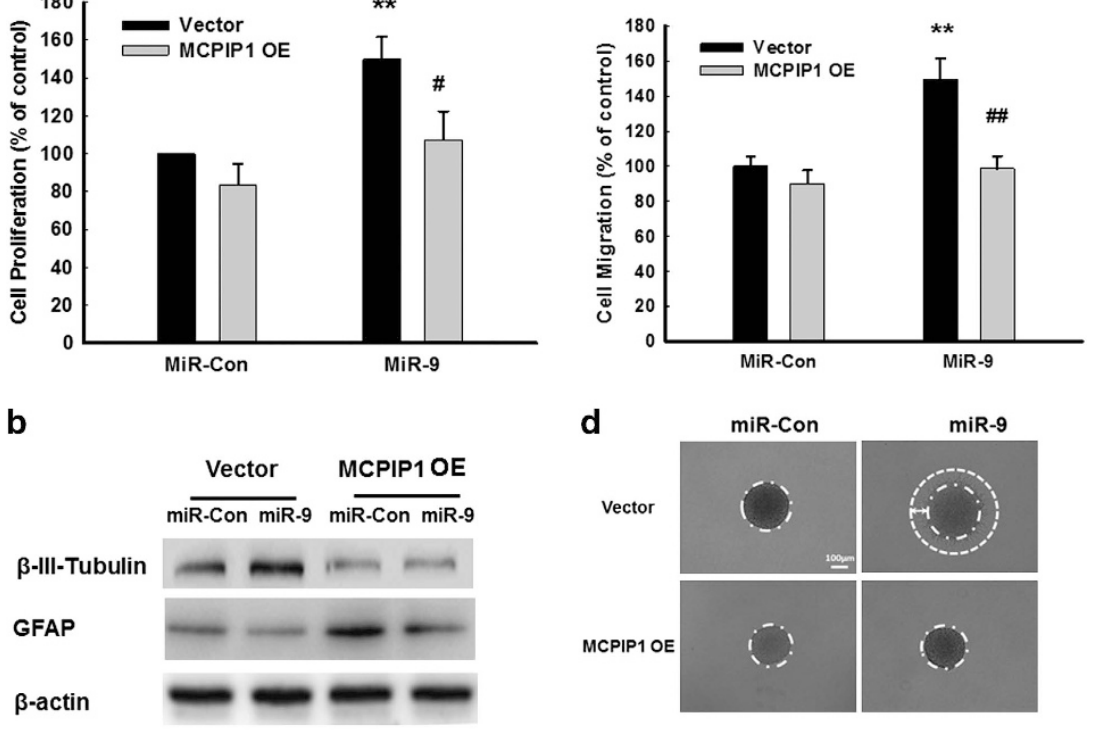

d

d miR-Con
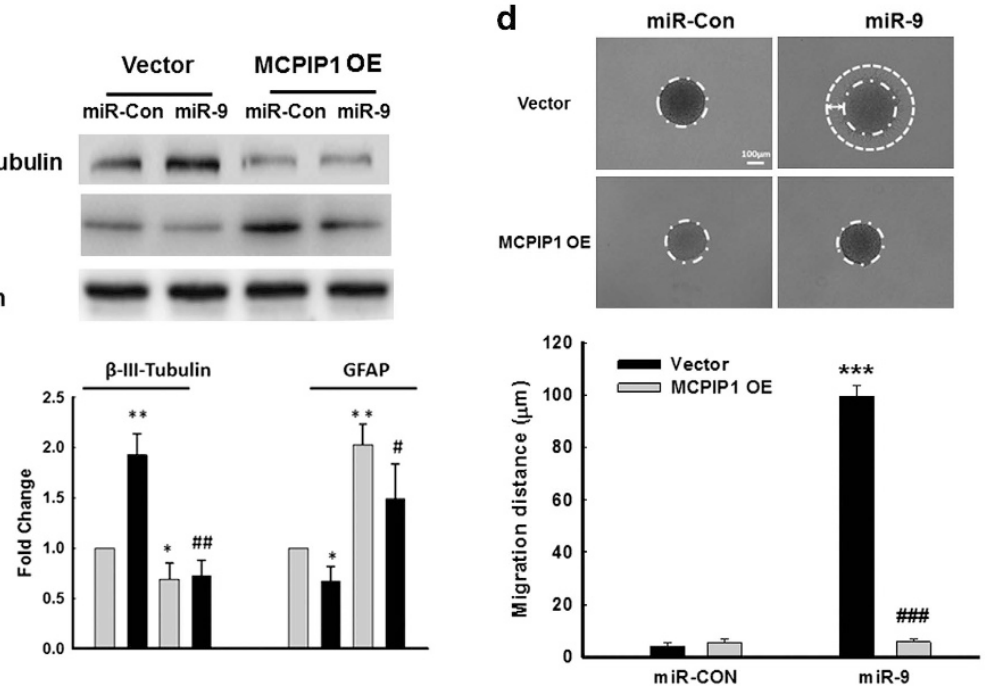

Figure 5 MCPIP1 overexpression antagonizes the effects of miR-9. (a) Transfection of primary NPCs with MCPIP1 OE-3'-UTR construct resulted in amelioration of miR9-mediated increase of NPC proliferation. (b) Transfection of primary NPCs with MCPIP1 OE-3'-UTR construct resulted in abrogation of miR-9-mediated increase of neuronal differentiation with a concomitantly opposing effect on GFAP expression. (c) Transfection of primary NPCs with MCPIP1 OE-3'-UTR construct resulted in abrogation of miR-9mediated increase in NPC migration using the Boyden chamber. (d) Transfection of primary NPCs with MCPIP1 OE-3'-UTR construct resulted in decrease in miR-9-mediated migration using the 3-D cell culture system. All the data are indicated as mean \pm S.D. of four individual experiments. ${ }^{*} P<0.05,{ }^{* *} P<0.01,{ }^{* * *} P<0.001$ versus NPCs co-transfected with vector/miR-control group; ${ }^{\#} P<0.05,{ }^{\# \#} P<0.01$, ${ }^{\# \# \#} P<0.001$ versus NPCs co-transfected with vector/miR-9 group

Although Akt inhibition significantly downregulated CREB expression, there was still residual activation of CREB, leading to the idea that other alternative pathways might be involved in the regulation of CREB activation by PDGF-BB.

Differential role of NF- $\kappa \mathrm{B}$ and CREB pathway in miR-9/ MCPIP1-mediated NPC proliferation, differentiation and migration. Previous study from our group has demonstrated that exposure of NPC to PDGF-BB resulted in increased proliferation through activation of $\mathrm{NF}-\kappa \mathrm{B}$ and $\mathrm{CREB}$ pathways. $^{7}$ The next logical step was to examine the functional relevance of NF- $\kappa \mathrm{B}$ and CREB in differentiation and migration of NPCs. Pretreatment of NPCs with IKK2 inhibitor SC514 $(5 \mu \mathrm{M})$ resulted in significant decrease in PDGF-BBmediated differentiation of NPCs (Figure 7a). This was further confirmed by the fact that transfection of NPCs with mutant $I_{\kappa} \mathrm{B}$ resulted in abrogation of PDGF-BB-mediated NPC differentiation (Figure $7 b$ ). To examine the role of CREB, cells were pretreated with the protein kinase A (PKA) inhibitor H89 $(5 \mu \mathrm{M})$ and assessed for differentiation of NPCs. As shown in Figure 7c, pre-treatment with the inhibitor resulted in significant decrease in PDGF-BB-mediated NPC differentiation. Interestingly, pre-treatment of NPCs with H89, but not SC514, inhibited PDGF-BB-induced NPC migration (Figure $7 \mathrm{~d}$ ). The role of NF- $\kappa$ B pathway in PDGF-BB-induced NPC migration was further confirmed by transfection cells with mutant $\mathrm{I}_{\kappa} \mathrm{B}$ (Figure 7e). Taken together, these data suggested that PDGF-BB/miR-9/MCPIP1/NF- $\kappa$ B pathway was involved in miR-9-mediated NPC proliferation and differentiation, whereas MCPIP1/CREB pathway regulated miR-9-mediated NPC proliferation, differentiation and migration.

\section{Discussion}

New dentate granule cells are continuously generated from neural progenitor cells and are integrated into the existing hippocampal circuitry in the adult mammalian brain through an orchestrated process termed as adult neurogenesis. ${ }^{1}$ Active neurogenesis occurs throughout life and relies upon the orchestrated processes involving proliferation, differentiation and migration and is regulated by a variety of pathological as well as physiological stimuli including neurotropic factors. ${ }^{21-24}$ Although the neurotropic factors are known to impact neurogenesis positively, ${ }^{25}$ the mechanism(s) underlying regulation by these factors of the specific miRNAs and their targets in this process remains less understood. 
a
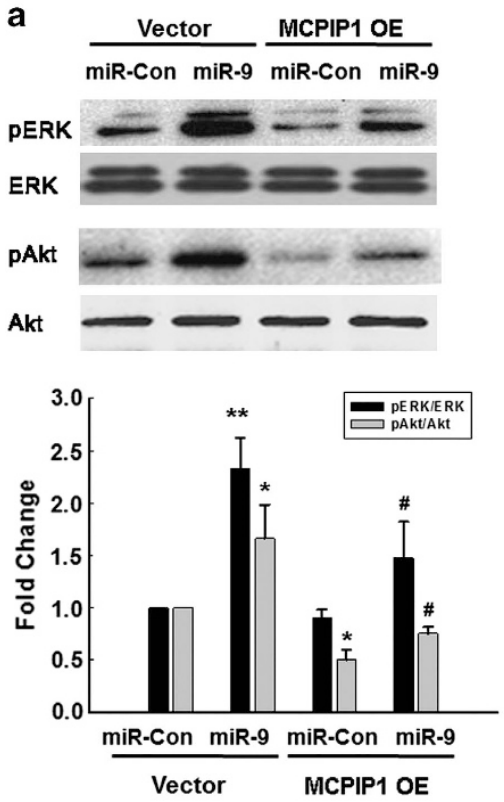

b
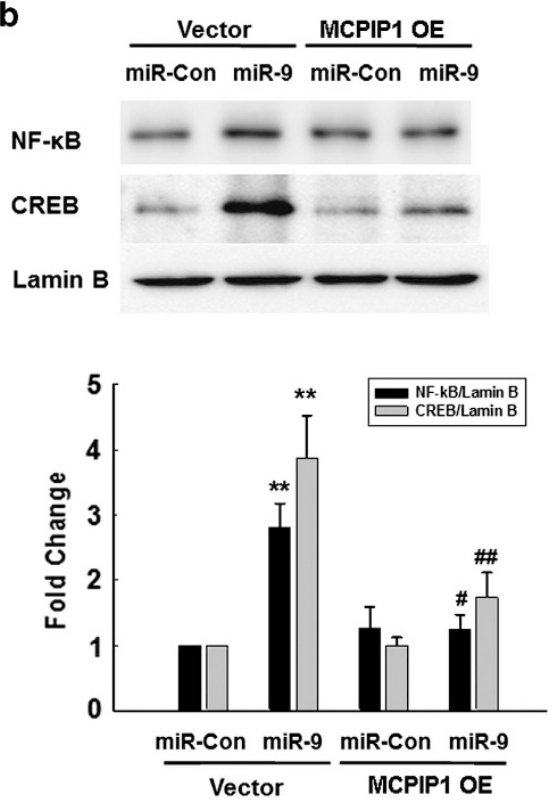

C
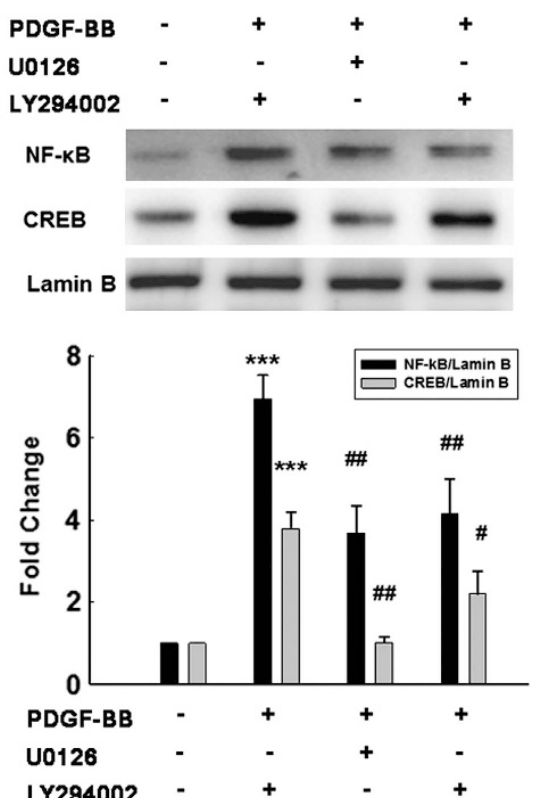

Figure 6 MCPIP1 overexpression abrogates miR-9-mediated signaling. (a) Transfection of cells with MCPIP1 OE-3'-UTR construct inhibited miR-9-mediated ERK and Akt phosphorylation. (b) Transfection of cells with MCPIP1 OE-3'-UTR construct inhibited nuclear translocation of NF- $k$ B and CREB. All the data are indicated as mean \pm S.D. of four individual experiments. ${ }^{*} P<0.05,{ }^{* \star} P<0.01$ versus NPCs co-transfected with vector/miR-control group; ${ }^{\#} P<0.05,{ }^{\# \#} P<0.01$ versus NPCs co-transfected with vector/ miR-9 group. (c) Pre-treatment of NPCs with MEK (U0126) or PI3K (LY294002) inhibitors abrogated PDGF-BB-mediated activation of both NF- $\kappa B$ and CREB signaling pathways. All the data are indicated as mean \pm S.D. of four individual experiments. ${ }^{* * \star} P<0.001$ versus NPCs in control group; ${ }^{\#} P<0.05,{ }^{\# \#} P<0.01$ versus NPCs treated with the PDGF-BB group

Herein, we provide new insights into the novel roles of miR-9/ MCPIP1 in PDGF-BB-mediated regulation of NPC proliferation, differentiation as well as migration via the common suppression of miR-9 target, MCPIP1. Furthermore, we demonstrated distinct downstream activation of NF- $\kappa \mathrm{B}$ in NPC proliferation and differentiation while the activation of CREB pathway in all of the neurogenesis processes. In recent times, much progress has been made in the development of therapeutic strategies for treatment of various neurodegenerative disorders. ${ }^{2,7,12}$ The findings on miR-9/MCPIP1 presented herein implicate it as a desirable target in PDGFBB-mediated cell signaling for regulation of neurogenesis, with implications treatment of neurodegenerative disorders associated with impaired neurogenesis.

Multiple lines of evidence have indicated that PDGF-BB has a key role in neurogenesis. ${ }^{2,7}$ For example, our previous studies have demonstrated PDGF-BB-mediated upregulation of NPC proliferation. In addition, it was also shown that PDGF-BB triggered differentiation of NPCs into neurons, and also had the potential to increase NPC migration. As miR-9 is a highly conserved microRNA that is predominantly expressed in the CNS of the developing embryo ${ }^{11}$ and that has a vital role in neurogenesis, we rationalized that PDGFBB-mediated regulation of neurogenesis ${ }^{12-15}$ could involve miR-9-mediated functions. Among the other functions of miR-9 in the CNS are its ability to mediate regulation of axonal extension and branching via targeting Map1b in the mouse cortical neurons. ${ }^{16}$ Recent studies have also implicated the role of miR-9 in regulating the stability of the notch target gene mRNA-Hes1, with implication in Hes1 ultradian oscillations. ${ }^{17}$ Although miR-9 is known to promote proliferation of neuronal progenitors by targeting stathimin, ${ }^{12}$ the role of relevant miRNA(s) and their target(s) critical in neurotropic factor (PDGF-BB)-mediated regulation of neurogenesis have not been identified thus far.

In this study, we demonstrate that exposure of NPCs to PDGF-BB resulted in increased expression of miR-9 by both real-time PCR and FISH assays (Figure 1), thus providing a biological basis for interaction between PDGF-BB and miR-9. Intriguingly, PDGF-BB-mediated upregulation of NPC proliferation $^{7}$ was abrogated in NPCs transduced with anti-miR-9 lentivirus construct, thereby implicating the role of miR-9 as a downstream mediator of PDGF-BB-mediated regulation of neurogenesis. Our findings on miR-9-mediated increase in NPC proliferation are in agreement with a previous report demonstrating a similar effect of miR-9 in human embryonic stem cell-derived progenitors. ${ }^{12}$ In concordance with the study of Zhao et al. ${ }^{15}$ that overexpression of miR-9 via suppression of the expression of nuclear receptor TLX was critical for neural stem cell fate determination, our results also suggest the role for miR-9 in neuronal differentiation and NPC migration. Similar to the study by these authors, we also observed increased migration of NPCs following miR-9 overexpression. ${ }^{15}$ Our findings however are in contrast to those published by Delaloy et al., ${ }^{12}$ wherein the authors demonstrate that loss of miR-9 promoted migration of multipotent hNPCs during the early neurosphere stage. The reason for this discrepancy could be attributed to the varying cell culture conditions and the source of cells. In our study, we used embryonic rat hippocampal neuronal progenitors instead of rat cortical or human embryonic stem cell-derived neuronal progenitors. ${ }^{12}$ Taking into account these findings, 
8
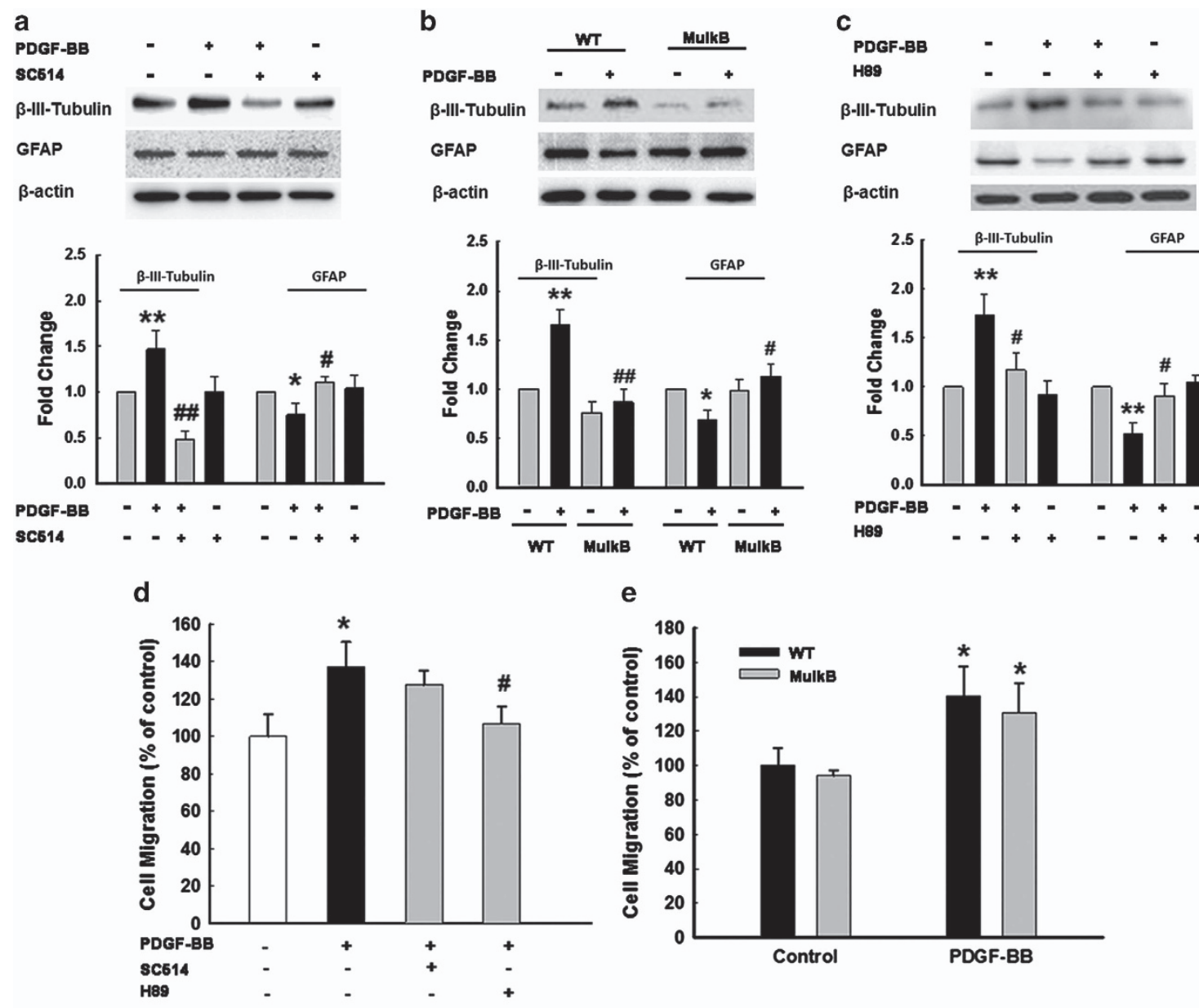

e
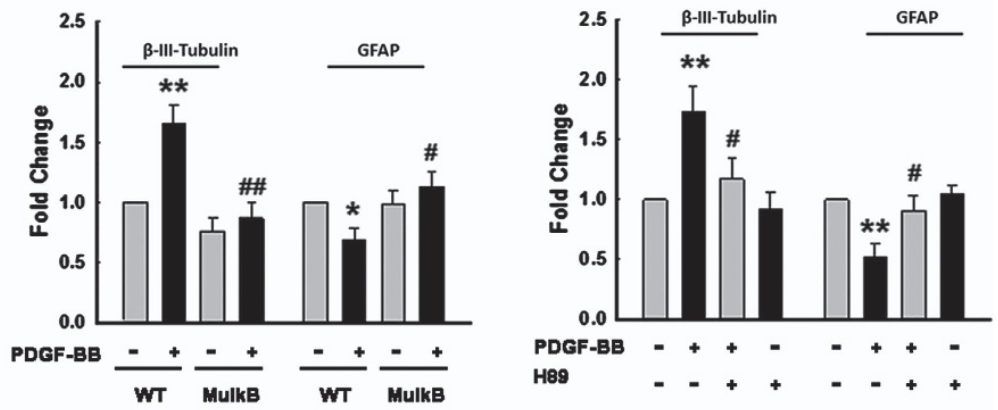

f

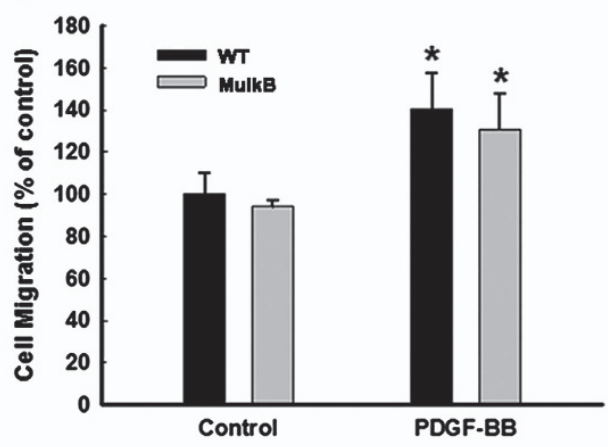

f
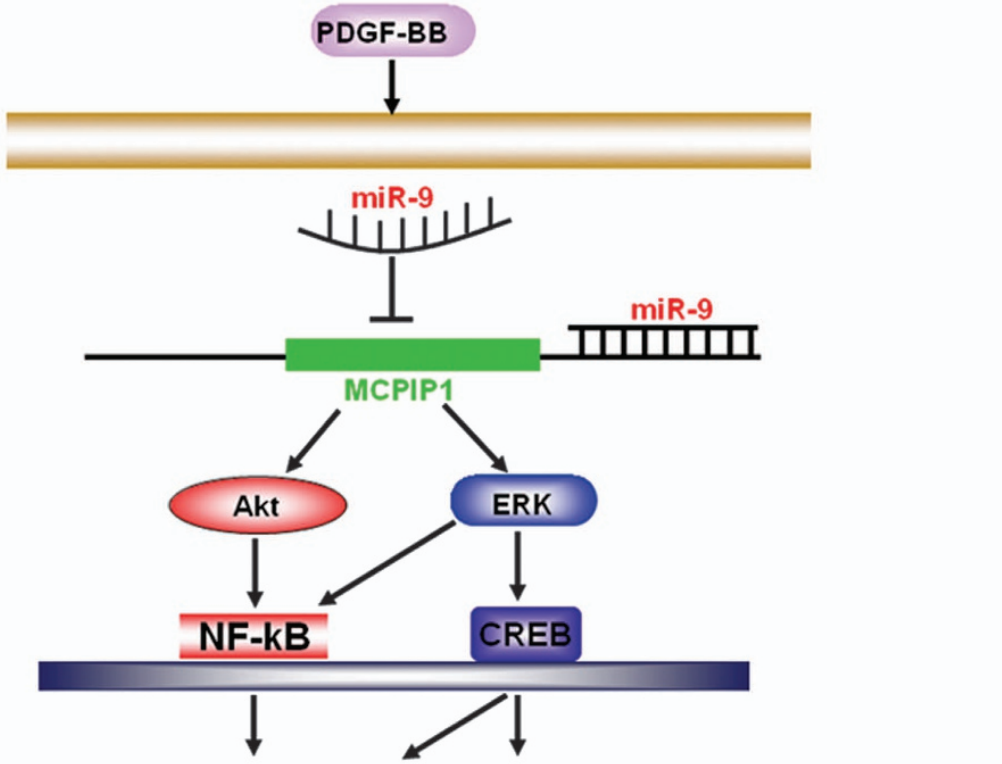

NPC Proliferation \&

NPC Migration Differentiation 
it can be speculated that the same miRNA has the capacity to display distinct functions during different stages of development of various NPCs, probably via differential spatial and temporal regulation of the target genes. This could lead to the speculation of yet another regulatory control mechanism by the miRNAs.

Another novel finding of this study was that MCPIP1, a newly identified molecule was a target of miR-9-mediated NPC proliferation, differentiation and migration. TargetScan algorithms and our experimental findings (unpublished data) identified MCPIP1 as the target of miR-9. In keeping with the function of miR-9 in PDGF-BB-mediated regulation of neurogenesis, and that MCPIP1 is a target of miR-9, transfection of NPCs with MCPIP1 OE-3'-UTR construct resulted in inhibition of PDGF-BB-mediated increase of NPC proliferation. Previous studies have demonstrated that MCPIP1 induced apoptosis in various cell types. ${ }^{18,26}$ However, in the current study we failed to detect the apoptotic effect of MCPIP1 overexpression in NPCs (data not shown), thereby ruling out the possibility that MCPIP1-mediated reduced NPC proliferation was due to apoptosis of cells. In addition to impacting NPC proliferation, MCPIP1 overexpression also reversed neuronal differentiation triggered by PDGF-BB. Thus, by inference these results lend credence to previous reports demonstrating the involvement of MCPIP1 signaling in upregulating glial differentiation of NT2 neuroprogenitor cells. ${ }^{20}$ Herein, we have also provided a novel role of MCPIP1 in cell migration as evidenced by the fact that transfection of NPCs with MCPIP1-overexpressing construct significantly inhibited PDGF-BB- or miR-9-induced NPC migration.

Following PDGF-BB stimulation of miR-9/MCPIP1, multiple signaling pathways are regulated and may intersect with different transcription factors. In this study, we showed that ERK MEKs and PI3K/Akt pathways are coregulated in NPCs cotransduced and transfected with miR-9 lentivirus/MCPIP1overexpressing constructs (Figure 5). Earlier reports have demonstrated that both ERK and PI3K/Akt pathways are required for NPC proliferation. ${ }^{27,28} \mathrm{MCPIP} 1$ is known to exert its action via inhibition of activation of NF- $\kappa \mathrm{B} .{ }^{29}$ Consistent with this finding, transduction of NPCs with miR-9 increased $\mathrm{NF}-\kappa \mathrm{B}$ translocation into the nucleus, which was reversed by transfection of cells with MCPIP1 OE $3^{\prime}$-UTR construct (Figure 6). Using both pharmacological and genetic approaches, we demonstrated that NF- $\kappa \mathrm{B}$ activation was critical for PDGF-BB-mediated NPC proliferation and neuronal differentiation but not in NPC migration. Our results are in agreement with previous reports demonstrating the role of NF $-\kappa$ B activation in NPC proliferation ${ }^{30}$ and differentiation. ${ }^{31}$ Although mounting evidence suggests that NF- $\kappa \mathrm{B}$ not only has a role in NPC proliferation but also is vital in the control of migration in stem cells treated with $\mathrm{MCP}-1,{ }^{32}$ our findings failed to detect the role of NF- $\kappa$ B in PDGF-BB-induced NPC migration. Overall these findings lead us to suggest that a common signal-transduction pathway such as NF- $\kappa \mathrm{B}$ could be critical in regulating diverse biological processes in the same cell type and also that it could also display other additional functions in other cell types.

Another interesting and novel finding herein was the observation that inhibition of CREB signaling resulted in abrogation of PDGF-BB-induced NPC proliferation, neuronal differentiation and migration. On the basis of these findings, it can be postulated that CREB that lies downstream of miR-9/ MCPIP1 has a critical role in PDGF-BB-mediated neurogenesis. Similar to NF- $\kappa$ B activation, transduction of NPCs with miR-9 increased CREB translocation into the nucleus, an effect that was reversed by transfection of cells with MCPIP1 OE $3^{\prime}$-UTR construct. Our findings are in agreement with a previous report demonstrating the role of CREB activation in NPC proliferation in the embryonic zebrafish. ${ }^{33}$ Similarly, another report has also demonstrated that reciprocally, inhibition of CREB signaling resulted in decreased NPC proliferation. ${ }^{34}$ In addition to the critical role in NPC proliferation, our data are also consistent with another earlier report that CREB activation played a role in neuronal differentiation in adult neural progenitors, ${ }^{28}$ as evidenced by the fact that PKA inhibitor H89 significantly decreased PDGF-BB-induced neuronal differentiation in NPCs (Figure 6). In addition to NPC proliferation and neuronal differentiation, our findings also underpin the role of CREB in NPC migration. Our results suggest that CREB signaling that lies downstream of miR-9/ MCPIP1 has a critical role in regulating NPC migration as MCPIP1 OE-3'-UTR in NPCs resulted in abrogation of miR-9induced CREB activation as well as NPC migration. This finding was consistent with the previous report that pharmacological blockade of CREB activation resulted in impairment of neuronal migration. ${ }^{35}$ As MCPIP1 was involved in the regulation of CREB activation, future elucidation of the relationship between these two pathways needs to be explored further.

In summary, miR-9 has a crucial role in NPC proliferation, neuronal differentiation and migration via suppression of MCPIP1 expression and subsequent downstream activation of NF- $\kappa \mathrm{B}$ and CREB pathways as summarized in Figure $7 f$. Taken together, our findings suggest that the two distinct pathways are critical for miR-9/MCPIP1-mediated signal transduction involved in proliferation and differentiation while CREB is uniquely critical for migration of NPCs. MiR-9/ MCPIP1 axis thus mediates a regulatory pathway critical for eliciting neurogenesis. Development of therapeutic strategies regulating miR-9/MCPIP1 axis could be considered as a potential target for the boosting neurogenesis in the treatment

Figure 7 Differential roles of NF- $\kappa$ B and CREB pathways in PDGF-BB-mediated NPC differentiation and migration. (a) Effect of I $\kappa$ B kinase 2 (IKK2) inhibitor SC514 on PDGF-BB-mediated NPC differentiation. (b) Effect of mutant $I_{\kappa} B$ on PDGF-BB-mediated NPC differentiation. NPCs were transfected with either WT or mutant $I_{\kappa} B$ plasmids for 4 days followed by assessment of NPC differentiation. (c) Effect of PKA inhibitor H89 on PDGF-BB-mediated NPC differentiation. (d) Pre-treatment of NPCs with SC514 but not $\mathrm{H} 89$ inhibitors abrogated PDGF-BB-mediated increase of NPC migration. (e) Effect of mutant $1 \kappa B$ on PDGF-BB-mediated NPC migration. All the data are indicated as mean \pm S.D. of four individual experiments. ${ }^{*} P<0.05$, ${ }^{\star \star} P<0.01$ versus control group; ${ }^{\sharp} P<0.05$, ${ }^{\# \#} P<0.01$ versus PDGF-BB-treated group. (f) Schematic illustration demonstrating the role of miR-9/MCPIP1 in PDGF-BB-mediated neurogenesis. PDGF-BB-mediated upregulation of miR-9 resulted in the activation of Akt and ERK pathways via suppression of the miR-9 target MCPIP1, with subsequent activation of NF- $\kappa B$ and CREB transcription factors. MiR-9-mediated NPC proliferation and neuronal differentiation involves both NF- $\kappa$ B and CREB pathways, whereas the NPC migration involves CREB but not the NF- $\kappa$ B pathway 
of various neurodegenerative disorders involving impaired neurogenesis.

\section{Materials and Methods}

Reagents. Recombinant PDGF-BB was purchased from R\&D Systems (Minneapolis, MN, USA). The specific PI3K inhibitor LY294002 and MEK1/2 inhibitor U0126 were purchased from Calbiochem (San Diego, CA, USA). IKK2 inhibitor SC514 was purchased from Sigma Chemicals (St. Louis, MO, USA). H89 was obtained from Tocris (Park Ellisville, MO, USA). The primary antibodies used were: p-ERK/ERK, p-Akt/Akt, p-CREB/CREB, p65NF- $\kappa B$, lamin B (Cell Signaling, Danvers, MA, USA; $1: 200$ ) and $\beta$-actin (Sigma; $1: 4000$ ).

Isolation, differentiation and characterization of NPCs. NPCs were derived from the hippocampus of embryonic day 18 fetus were cultured in substrate-free tissue culture T75 flasks as reported previously by Tian et al. ${ }^{7,36}$ and our previous study. ${ }^{7}$ NPCs were treated with PDGF-BB $(100 \mathrm{ng} / \mathrm{ml}){ }^{7}$ Treatment of NPCs with pharmacological inhibitors (U0126: $20 \mu \mathrm{M}$; LY294002: $20 \mu \mathrm{M}$; SC514: $5 \mu \mathrm{M}$; H89: $5 \mu \mathrm{M}$ ) involved pre-treating cells with the respective inhibitors for $1 \mathrm{~h}$ followed by exposure with PDGF-BB. Forty-eight hours later, cells were examined for cell proliferation.

Cell proliferation. Cell proliferation was measured by CyQUANT cell proliferation assay according to the manufacture's protocol (Invitrogen, Grand Island, NY, USA) and our previous report. ${ }^{7}$ NPCs dissociated from neurosphere with/without transduction or transfection with lentivirus miRNAs or plasmids were seeded in 96-well plates at a density of $10^{4}$ cells per well for 2 days and were treated with PDGF-BB for $24 \mathrm{~h}$. Then, $200 \mu \mathrm{l}$ of the CyQUANT GR dye/cell lysis buffer was added into each well and incubated in the $\mathrm{CO}_{2}$ incubator for $15 \mathrm{~min}$. Fluorescence intensity of each well was obtained using a Dynatech MR5000 plate counter (Chantilly, VA, USA) at excitation and emission wavelengths of 480 and $520 \mathrm{~nm}$, respectively.

Immunocytochemistry. For immunocytochemistry, NPCs were plated onto coverslips, fixed with $4 \%$ paraformaldehyde and permeabilized with $0.3 \%$ Triton $\mathrm{X}-100$ in phosphate-buffered saline (PBS). Cells were then incubated with a blocking buffer followed by incubation with mouse anti- $\beta$-III-tubulin (1:500; Sigma) and mouse anti-GFAP (1:1000; Sigma) antibodies overnight at $4{ }^{\circ} \mathrm{C}$. Secondary AlexaFluor 594 goat anti-mouse IgG was added at a 1:500 dilution for $2 \mathrm{~h}$ to detect $\beta$-III-tubulin and GFAP, followed by mounting of cells with Vectashield onto glass slides (Vector Laboratories, Burlingame, CA, USA). Fluorescent images were acquired at room temperature (RT) on a Zeiss Observer. A Z1 inverted microscope (Carl Zeiss, Thornwood, NY, USA) was used; images were processed using AxioVs 40 Version 4.8.0.0 software (Carl Zeiss Microlmaging $\mathrm{GmbH}$ ). Photographs were acquired using an AxioCam MRm digital camera (Carl Zeiss).

Plasmid transfection. NPCs were transfected with Flag/Flag-MCPIP1, wild type (WT)/mutant I $\kappa$ B plasmids using the rat stem cell Nucleofector Kit (Amaxa, Gaithersburg, MD, USA) according to the manufacturer's instructions. Briefly, dissociated cells from neurospheres were resuspended in the transfection medium, mixed with respective plasmids ( $2.5 \mu \mathrm{g}$ per well, 24-well plate) and electroporated following which cells were quickly centrifuged, resuspended and plated. Cells were treated with PDGF-BB and examined for proliferation, differentiation or migration at $96 \mathrm{~h}$ following transfections.

Western blotting. Treated cells were lysed using the Mammalian Cell Lysis Kit (Sigma-Aldrich, St Louis, MO, USA). Equal amounts of the proteins were electrophoresed in a sodium dodecyl sulfate-polyacrylamide gel (12\%) under reducing conditions, followed by transfer to polyvinylidine fluoride membranes. The blots were blocked with $5 \%$ non-fat dry milk in PBS. The western blots were then probed with antibodies recognizing the MCPIP1 (Santa Cruz, Dallas, TX, USA), ERK, Akt, p65 NF- $k$ B and CREB (1:200; Cell Signaling). The secondary antibodies were alkaline phosphatase conjugated to goat anti-mouse/rabbit lgG $(1: 5000)$ as described previously. ${ }^{37}$

Real-time PCR. For quantitative analysis of mRNA expression, comparative real-time PCR was performed with the use of the SYBR Green PCR Master Mix (Applied Biosystems, Grand Island, NY, USA). The sequences for the amplification of rat MCPIP were: $5^{\prime}$-TGCCTATCACAGACCAGCAC-3' (forward) and 5'-TGT CATTGGACACCACCACTC-3' (reverse). The sequences for the amplification of rat pre-miR-9-1 were: $5^{\prime}$-CAGAGAAGGGCAGTGGAGAC-3' (forward) and 5'-ACG ACAGAGACCGAAAAAGG-3' (reverse); pre-miR-9-2 were: 5'-CTAACGCTGCCG GAGATTAC-3' (forward) and 5'-TACTTGCCGCGCTTAAGATT-3' (reverse); pre-miR-9-3 were: $5^{\prime}$-GCGCTCGAGGCTCTCTAAG-3' (forward) and 5'-GAGGG GATGGACAGACACAC- $3^{\prime}$ (reverse). The primer sequences for the amplification of GAPDH were as follows: $5^{\prime}$-GCCAAAAGGGTCATCATCTC-3' (forward); $5^{\prime}$-GGCATGGACTGTGGTCATGAG-3' (reverse). For the analysis of miR-9, total RNA was isolated from cells as described above. Comparative real-time PCR was performed using the Taqman Universal PCR Master Mix (Applied Biosystems). Specific primers and probes for mature miR-9 and snRNA RNU6B were obtained from Applied Biosystems. All reactions were run in triplicate. The amount of miR-9 was obtained by normalizing to snRNA RNU6B and relative to control as reported previously. ${ }^{38}$

In situ hybridization and immunostaining. Primary NPCs (d12 in vitro) were fixed and were prehybridized in hybridization buffer ( $50 \%$ formamide, $10 \mathrm{mM}$ Tris- $\mathrm{HCl}$, pH 8.0, $200 \mu \mathrm{g} / \mathrm{ml}$ yeast tRNA, $1 \times$ Denhardt's solution, $600 \mathrm{mM}$ $\mathrm{NaCl}, 0.25 \%$ SDS, $1 \mathrm{mM}$ EDTA, $10 \%$ dextran sulfate) at a concentration of $9 \mathrm{pM}$ for the commercially available digoxigenin-labeled miR-9 probe (Exiqon, Woburn, MA). LNA-modified miR-9, labeled at both the $5^{\prime}$ and $3^{\prime}$ ends with digoxigenin (Exiqon), were diluted to a final concentration of $2 \mathrm{pM}$ in hybridization buffer, heated to $65^{\circ} \mathrm{C}$ for $5 \mathrm{~min}$ and separately hybridized to the sections at $37^{\circ} \mathrm{C}$ overnight. The slides were then washed two times in hybridization buffer (without probe) at $37^{\circ} \mathrm{C}$, followed by washing three times in $2 \times$ saline-sodium citrate (SSC) and two times in $0.2 \times \mathrm{SSC}$ at $42^{\circ} \mathrm{C}$. They were then blocked with $1 \%$ bovine serum albumin, $3 \%$ normal goat serum in $1 \times$ PBS for $1 \mathrm{~h}$ at RT and incubated with antidigoxigenin conjugated with horseradish peroxidase (1:100; Roche Diagnostics $\mathrm{GmbH}$, Mannheim, Germany) and rat anti-nestin (1:100; Millpore, Billerica, MA, USA) antibodies overnight at $4^{\circ} \mathrm{C}$. The slides were washed two times with PBS and incubated with Alexa Fluor 488 goat anti-rat $\lg G(1: 400$; Invitrogen) antibody for $1 \mathrm{~h}$ at RT. This was followed by two PBS washes and signal amplification (for the in situ, now labeled with horseradish peroxidase) using TSA Cy5 kit (Perkin-Elmer, Waltham, MA, USA) according to the manufacturer's protocol. The slides were mounted in Prolong gold anti-fade reagent with 4',6-diamidino-2-phenylindole (Invitrogen). The specificity of the miR-9 signal in FISH experiments was confirmed when compared with a scrambled control. Unlike the miR-9, the scramble probe showed no signal in NPCs.

Transduction of primary NPCs with miR-9 or anti-miR-9 lentivirus. Primary NPCs were transduced with miR-control, miR-9, anti-miRcontrol, anti-miR-9 lentivirus with multiplicity of infection $=100$, followed by gentle swirling, incubation and replacement of fresh feed medium. Cells will be used for experiments at four days post-transduction.

MiR-9 increased NPC migration in vitro. Migration of NPCs in vitro was determined using Boyden Chamber (Millipore, Billerica, MA, USA) modified according the previous report and our previous studies. ${ }^{39-41}$ Cells transduced with miR-control or miR-9 were washed with PBS and fluorescently labeled with $10 \mu \mathrm{M}$ cell Tracker Green (Molecular Probes, Grand Island, NY, USA) for $10 \mathrm{~min}$ at RT. Labeled cells $\left(2 \times 10^{5}\right.$ cells) were added to the upper compartment of transwell inserts in serum-free medium. The transwell plates were incubated for $3 \mathrm{~h}$ at $37^{\circ} \mathrm{C}$ followed by quantification of NPC migration by measuring the number of migrated cells following detachment of cells from the insert using a Synergy Mx fluorescence plate reader (BioTek Instruments, Winooski, VT, USA).

3-D cell migration assay. The collagen matrix model was utilized as described previously. ${ }^{42-44}$ Final matrix parameters were: volume $=0.2 \mathrm{ml}$; diameter $=12 \mathrm{~mm}$; collagen concentration $=1.5 \mathrm{mg} / \mathrm{ml}$; and cell concentration $=10-20$ neurosphere/matrix. Matrices were established in 24-well plates (BD; no.353047), and incubated with neuronal progenitor basal medium. Cell migration was examined by measuring the distance from the edge of the neurosphere to the nucleus of the most distant cell according the previous report. ${ }^{12}$

Statistical analysis. Data were expressed as mean \pm standard deviation (S.D.). Significance of differences between control and samples treated with various drugs was determined by one-way ANOVA followed by post hoc least significant difference test. Values of $P<0.05$ were taken as statistically significant. 


\section{Conflict of Interest}

The authors declare no conflict of interest.

Acknowledgements. This work was supported by Grants MH-068212, DA020392, DA023397, DA024442 (SB) from the National Institutes of Health in addition to Nebraska Tobacco Settlement Biomedical Research.

1. Venkatesan A, Nath A, Ming GL, Song H. Adult hippocampal neurogenesis: regulation by HIV and drugs of abuse. Cell Mol Life Sci 2007; 64: 2120-2132.

2. Mohapel $P$, Frielingsdorf $H$, Haggblad J, Zachrisson O, Brundin P. Platelet-derived growth factor (PDGF-BB) and brain-derived neurotrophic factor (BDNF) induce striata neurogenesis in adult rats with 6-hydroxydopamine lesions. Neuroscience 2005; 132 : 767-776.

3. Almeida RD, Manadas BJ, Melo CV, Gomes JR, Mendes CS, Graos MM et al. Neuroprotection by BDNF against glutamate-induced apoptotic cell death is mediated by ERK and PI3-kinase pathways. Cell Death Differ 2005; 12: 1329-1343.

4. Bergsten E, Uutela M, Li X, Pietras K, Ostman A, Heldin CH et al. PDGF-D is a specific protease-activated ligand for the PDGF beta-receptor. Nat Cell Biol 2001; 3: 512-516.

5. Heldin $\mathrm{CH}$, Eriksson U, Ostman A. New members of the platelet-derived growth factor family of mitogens. Arch Biochem Biophys 2002; 398: 284-290.

6. Li X, Ponten A, Aase K, Karlsson L, Abramsson A, Uutela M et al. PDGF-C is a new protease-activated ligand for the PDGF alpha-receptor. Nat Cell Biol 2000; 2: 302-309.

7. Yao H, Duan M, Yang L, Buch S. Platelet-derived growth factor-BB restores human immunodeficiency virus Tat-cocaine-mediated impairment of neurogenesis: role of TRPC channels. J Neurosci 2012; 32: 9835-9847.

8. Fineberg SK, Kosik KS, Davidson BL. MicroRNAs potentiate neural development Neuron 2009; 64: 303-309.

9. Luikart BW, Perederiy JV, Westbrook GL. Dentate gyrus neurogenesis, integration and microRNAs. Behav Brain Res 2012; 227: 348-355.

10. Shi Y, Zhao X, Hsieh J, Wichterle H, Impey S, Banerjee S et al. MicroRNA regulation of neural stem cells and neurogenesis. J Neurosci 2010; 30: 14931-14936.

11. Wienholds E, Kloosterman WP, Miska E, Alvarez-Saavedra E, Berezikov E, de Bruijn E et al. MicroRNA expression in zebrafish embryonic development. Science 2005; 309: 310-311.

12. Delaloy C, Liu L, Lee JA, Su H, Shen F, Yang GY et al. MicroRNA-9 coordinates proliferation and migration of human embryonic stem cell-derived neural progenitors Cell Stem Cell 2010; 6: 323-335.

13. Leucht C, Stigloher C, Wizenmann A, Klafke R, Folchert A, Bally-Cuif L. MicroRNA-9 directs late organizer activity of the midbrain-hindbrain boundary. Nat Neurosci 2008; 11 : 641-648.

14. Shibata M, Nakao H, Kiyonari H, Abe T, Aizawa S. MicroRNA-9 regulates neurogenesis in mouse telencephalon by targeting multiple transcription factors. J Neurosci $2011 ; 31$ 3407-3422.

15. Zhao C, Sun G, Li S, Shi Y. A feedback regulatory loop involving microRNA-9 and nuclear receptor TLX in neural stem cell fate determination. Nat Struct Mol Biol 2009; 16 365-371.

16. Dajas-Bailador F, Bonev B, Garcez P, Stanley P, Guillemot F, Papalopulu N. MicroRNA-9 regulates axon extension and branching by targeting Map1b in mouse cortical neurons. Nat Neurosci 2012; 15: 697-699.

17. Bonev B, Stanley P, Papalopulu N. MicroRNA-9 modulates Hes1 ultradian oscillations by forming a double-negative feedback loop. Cell Rep 2012; 2: 10-18.

18. Zhou L, Azfer A, Niu J, Graham S, Choudhury M, Adamski FM et al. Monocyte chemoattractant protein-1 induces a novel transcription factor that causes cardiac myocyte apoptosis and ventricular dysfunction. Circ Res 2006; 98: 1177-1185.

19. Liang J, Wang J, Azfer A, Song W, Tromp G, Kolattukudy PE et al. A novel CCCH-zinc finger protein family regulates proinflammatory activation of macrophages. J Biol Chem 2008; 283: 6337-6346.

20. Vrotsos EG, Kolattukudy PE, Sugaya K. MCP-1 involvement in glial differentiation of neuroprogenitor cells through APP signaling. Brain Res Bull 2009; 79: 97-103.

21. Wang H, Hughes I, Planer W, Parsadanian A, Grider JR, Vohra BP et al. The timing and location of glial cell line-derived neurotrophic factor expression determine enteric nervous system structure and function. J Neurosci 2010; 30: 1523-1538.

22. Bergami M, Rimondini R, Santi S, Blum R, Gotz M, Canossa M. Deletion of TrkB in adult progenitors alters newborn neuron integration into hippocampal circuits and increases anxiety-like behavior. Proc Natl Acad Sci USA 2008; 105: 15570-15575.
23. Yuan TF. BDNF signaling during olfactory bulb neurogenesis. J Neurosci 2008; 28 : 5139-5140.

24. Pinnock SB, Herbert J. Brain-derived neurotropic factor and neurogenesis in the adult rat dentate gyrus: interactions with corticosterone. Eur J Neurosci 2008; 27: 2493-2500.

25. Cuddon P Bootman MD, Richards GR, Smith AJ, Simpson PB, Roderick HL. Methacholine and PDGF activate store-operated calcium entry in neuronal precursor cells via distinct calcium entry channels. Biol Res 2008; 41: 183-195.

26. Hausmann EH, Berman NE, Wang YY, Meara JB, Wood GW, Klein RM. Selective chemokine mRNA expression following brain injury. Brain Res 1998; 788: 49-59.

27. Learish RD, Bruss MD, Haak-Frendscho M. Inhibition of mitogen-activated protein kinase kinase blocks proliferation of neural progenitor cells. Brain Res Dev Brain Res 2000; 122 : $97-109$

28. Peltier J, O'Neill A, Schaffer DV. PI3K/Akt and CREB regulate adult neural hippocampal progenitor proliferation and differentiation. Dev Neurobiol 2007; 67: 1348-1361.

29. Liang J, Saad Y, Lei T, Wang J, Qi D, Yang Q et al. MCP-induced protein 1 deubiquitinates TRAF proteins and negatively regulates JNK and NF-kappaB signaling. J Exp Med 2010; 207: 2959-2973.

30. Widera D, Mikenberg I, Kaltschmidt B, Kaltschmidt C. Potential role of NF-kappaB in adult neural stem cells: the underrated steersman? Int J Dev Neurosci 2006; 24: 91-102.

31. Feng Z, Porter AG. NF-kappaB/Rel proteins are required for neuronal differentiation of SH-SY5Y neuroblastoma cells. J Biol Chem 1999; 274: 30341-30344.

32. Widera D, Holtkamp W, Entschladen F, Niggemann B, Zanker K, Kaltschmidt B et al. MCP-1 induces migration of adult neural stem cells. Eur J Cell Biol 2004; 83: 381-387.

33. Dworkin S, Malaterre J, Hollande F, Darcy PK, Ramsay RG, Mantamadiotis T. cAMP response element binding protein is required for mouse neural progenitor cell survival and expansion. Stem Cells 2009; 27: 1347-1357.

34. Luo CX, Jin X, Cao CC, Zhu MM, Wang B, Chang $L$ et al. Bidirectional regulation of neurogenesis by neuronal nitric oxide synthase derived from neurons and neural stem cells. Stem Cells 2010; 28: 2041-2052.

35. Chiaramello S, Dalmasso G, Bezin L, Marcel D, Jourdan F, Peretto P et al. BDNF/TrkB interaction regulates migration of SVZ precursor cells via $\mathrm{PI} 3-\mathrm{K}$ and MAP-K signalling pathways. Eur J Neurosci 2007; 26: 1780-1790.

36. Tian C, Murrin LC, Zheng JC. Mitochondrial fragmentation is involved in methamphetamine-induced cell death in rat hippocampal neural progenitor cells. PLOS One 2009; 4 e5546.

37. Yao H, Peng F, Dhillon N, Callen S, Bokhari S, Stehno-Bittel L et al. Involvement of TRPC channels in CCL2-mediated neuroprotection against tat toxicity. J Neurosci 2009; 29 : 1657-1669.

38. Hu G, Zhou R, Liu J, Gong AY, Chen XM. MicroRNA-98 and let-7 regulate expression of suppressor of cytokine signaling 4 in biliary epithelial cells in response to Cryptosporidium parvum infection. J Infect Dis 2010; 202: 125-135.

39. Douglas-Escobar M, Rossignol C, Steindler D, Zheng T, Weiss MD. Neurotrophin-induced migration and neuronal differentiation of multipotent astrocytic stem cells in vitro. PLoS One 2012; 7: e51706.

40. Yao H, Duan M, Yang L, Buch S. Nonmuscle myosin light-chain kinase mediates microglial migration induced by HIV Tat: involvement of beta1 integrins. FASEB $J$ 2013; 27: $1532-1548$

41. Yao H, Yang Y, Kim KJ, Bethel-Brown C, Gong N, Funa K et al. Molecular mechanisms involving sigma receptor-mediated induction of MCP-1: implication for increased monocyte transmigration. Blood 2010; 115: 4951-4962.

42. Grinnell F, Zhu M, Carlson MA, Abrams JM. Release of mechanical tension triggers apoptosis of human fibroblasts in a model of regressing granulation tissue. Exp Cell Res 1999; 248: 608-619.

43. Lund AW, Stegemann JP, Plopper GE. Mesenchymal stem cells sense three dimensional type I collagen through discoidin domain receptor 1. Open Stem Cell J 2009; 1: 40-53.

44. Watanabe K, Nakamura M, Okano H, Toyama Y. Establishment of three-dimensional culture of neural stem/progenitor cells in collagen Type-1 gel. Restor Neurol Neurosci 2007; 25: 109-117.

(i) $\Theta$ Cell Death and Disease is an open-access journal published by Nature Publishing Group. This work is licensed under a Creative Commons Attribution-NonCommercialNoDerivs 3.0 Unported License. To view a copy of this license, visit http://creativecommons.org/licenses/by-nc-nd/3.0/ 